\title{
Prescribe an SGLT2 inhibitor for heart failure in the absence of diabetes?
}

\section{An RCT demonstrates that dapagliflozin produces better cardiovascular outcomes than placebo for heart failure patients with and without diabetes.}

\section{PRACTICE CHANGER}

Prescribe dapagliflozin, a sodium-glucose cotransporter-2 (SGLT2) inhibitor, $10 \mathrm{mg} / \mathrm{d}$ in addition to standard therapies for adult patients with heart failure (HF) with a reduced ejection fraction $(\leq 40 \%)$ and New York Heart Association (NYHA) class II or greater, regardless of type 2 diabetes history, due to improved heart failure and cardiovascular outcomes. ${ }^{1}$

STRENGTH OF RECOMMENDATION

B: Based on a single randomized controlled trial. ${ }^{1}$

McMurray JJV, Solomon SD, Inzucchi SE, et al. Dapagliflozin in patients with heart failure and reduced ejection fraction. $N$ Engl $J$ Med. 2019;381:1995-2008.

\section{ILLUSTRATIVE CASE}

A 64-year-old overweight White man with a history of hypertension, hyperlipidemia, and HF with an ejection fraction (EF) of $40 \%$ presents for primary care follow-up after a recent inpatient admission for worsened HF symptoms. At baseline, he is comfortable at rest but becomes dyspneic upon walking to another room within his home. He is already taking a mineralocorticoid receptor antagonist, a high-intensity statin, a beta-blocker, and an angiotensin-converting enzyme (ACE) inhibitor. What other medication should be considered to minimize his cardiovascular (CV) risk?
$\Lambda$ n estimated $1 \%$ to $2 \%$ of the world's adult population has HF. ${ }^{2}$ Although the exact prevalence is difficult to quantify due to variations in definitions and diagnostic methods, the American Heart Association (AHA) estimated that 6.2 million Americans had HF between 2013 and 2016. ${ }^{3}$ Prevalence increases with age, with an annual incidence of approximately 35 per 1000 by age $85 .{ }^{4}$ Due to the significant morbidity and mortality associated with HF, advancements in treatment are needed.

SGLT2 inhibitors work within the proximal tubule of the kidneys, resulting in increased glucose and sodium excretion with secondary osmotic diuresis and therefore a modest reduction in serum glucose. ${ }^{1,2,5,6}$ SGLT2 inhibitors are classically prescribed for hyperglycemia treatment in type 2 diabetes. However, preliminary data suggest that this class of medication also positively impacts cardiac function. The diuresis and natriuresis effects of SGLT2 inhibitors appear to optimize cardiac output and subsequent oxygen consumption through a reduction of afterload and preload. ${ }^{1,2,5,6}$ Further, SGLT2 inhibitors may decrease inflammatory pathways and lead to a secondary reduction of cardiac remodeling via a reduction and modulation of inflammatory pathways. This reduction and modulation may also be associated with a reduction in development, and possibly a reversal, of hypertrophic cardiomyopathy,

\author{
Debra Koenigsberger, \\ MD; Alexander Marquez, \\ MD; Pamela R. Hughes, \\ MD \\ Nellis AFB Family Medicine \\ Residency, Las Vegas, NV \\ DEPUTY EDITOR \\ Rebecca Mullen, \\ MD, MPH \\ University of Colorado \\ Department of Family \\ Medicine, Denver
}

doi: 10.12788/jfp.0235 


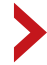

Dapagliflozin
demonstrated
decreased HF
exacerbations
and CV deaths,
improved
patient-reported
HF symptoms,
and lower all-
cause mortality
in patients
both with
and without
diabetes.

\section{Dapagliflozin}

decrear

exacerbations

and CV deaths

improved

patient-reported

oms,

in patients

diabetes. cardiac fibrosis, and atherosclerosis., ${ }^{5,6}$ Some of the previously reported adverse effects of SGLT2 inhibitors include urinary tract infection, acute kidney injury, lower extremity amputation, bone fracture, and diabetic ketoacidosis. $^{2}$

In several studies of patients with type 2 diabetes, SGLT2 inhibitors have shown benefit in reducing $\mathrm{CV}$ disease-related death and hospitalization for $\mathrm{HF}^{1,2,5,6} \mathrm{~A}$ recent expert consensus from the American College of Cardiology (ACC) states that SGLT2 therapy should be considered for any patient with type 2 diabetes who also has established atherosclerotic $\mathrm{CV}$ disease, $\mathrm{HF}$ (a clinical syndrome as defined in ACC/AHA guidelines), or diabetic kidney disease, or who is at a high risk for atherosclerotic CV disease (ie, has signs of end-organ damage, such as left ventricular hypertrophy or retinopathy, or multiple risk factors such as advanced age, smoking, hypertension, and family history).,

Additionally, a 2019 randomized controlled trial (RCT) by Nassif et al showed that, compared to placebo, dapagliflozin significantly improved both patient-reported HF symptoms and cardiac natriuretic peptide levels over 12 weeks in patients with and without diabetes. ${ }^{9}$ In September 2020, UpToDate added SGLT2 inhibitors as an option for patients with continued symptoms of HF despite use of appropriate primary agents and mineralocorticoid receptor antagonists, whether or not they have type 2 diabetes; this update was based on 2 studies, 1 of which is reviewed here. ${ }^{10}$

\section{STUDY SUMMARY}

\section{Dapagliflozin demonstrated better CV outcomes than placebo}

The Dapagliflozin and Prevention of Adverse Outcomes in Heart Failure (DAPA-HF) study is an RCT that compared dapagliflozin to placebo among 4744 patients ages 18 years and older who had HF with an $\mathrm{EF} \leq 40 \%$ and NYHA class II, III, or IV symptoms. The study included patients with (41.8\%) and without diabetes. Most patients were male (76.2\%-77\%), White (70\%), and European (44.7\%-46.1\%).

Patients were randomized to receive either dapagliflozin $10 \mathrm{mg} / \mathrm{d}$ or a matching placebo in addition to standard HF therapy (including an ACE inhibitor, angiotensin receptor blocker, or sacubitril-valsartan plus a beta-blocker unless contraindicated; mineralocorticoid antagonist use was encouraged). Follow-up occurred at 14 days, 60 days, 4 months, and then every 4 months, for an average of about 18 months. Patients with diabetes continued to use their glucose-lowering therapies, with dose adjustments, as needed.

The primary outcome was a composite of worsening HF (hospitalization or urgent visit requiring intravenous HF therapy) or death from a CV cause. Secondary outcomes included a composite of hospitalization for HF or CV death; total number of hospitalizations for $\mathrm{HF}$ (including repeat admissions) and CV death; a change in Kansas City Cardiomyopathy Questionnaire symptom score; a composite of worsening renal function including a sustained ( $\geq 28 \mathrm{~d}$ ) decline in the estimated glomerular filtration rate (eGFR) of $\geq 50 \%$, end-stage renal disease (defined as sustained eGFR of $<15 \mathrm{~mL} / \mathrm{min} / 1.73 \mathrm{~m}^{2}$, sustained dialysis, or renal transplantation), or renal death; and death from any cause.

The primary outcome of worsening HF or death from CV causes occurred in 386 of 2373 patients $(16.3 \%)$ in the dapagliflozin group and in 502 of 2371 patients $(21.2 \%)$ in the placebo group (hazard ratio $[\mathrm{HR}]=0.74$; 95\% CI, 0.65-0.85; $P<.001)$. The composite score of hospitalizations for HF plus death from a CV cause was lower in the dapagliflozin group compared to the placebo group (HR $=0.75 ; 95 \% \mathrm{CI}, 0.65-0.85 ; P<.001)$.

A total of 276 patients $(11.6 \%)$ in the dapagliflozin group and 329 patients (13.9\%) in the placebo group died from any cause ( $\mathrm{HR}=0.83$; 95\% CI, 0.71-0.97). More patients in the dapagliflozin group than in the placebo group had an improvement in symptom score $(58.3 \%$ vs $50.9 \%$; odds ratio $=1.15$; 95\% CI, 1.08-1.23; $P<.001)$. Renal composite outcome did not differ between the 2 treatment groups. Potential adverse effects included volume depletion, renal adverse event, and major hypoglycemia, which occurred at the same rate in the treatment and placebo groups. There was no difference in outcomes or adverse effects between patients with and without diabetes. ${ }^{1}$ 


\section{WHAT'S NEW}

\section{Evidence supports dapagliflozin use in a new patient population}

The DAPA-HF study compared dapagliflozin to placebo in HF patients both with and without diabetes and demonstrated decreased HF exacerbations and CV deaths, improved patient-reported HF symptoms, and lower all-cause mortality in the treatment group. This study supports use of dapagliflozin in a new patient population-those with HFrather than solely in patients with diabetes, as the drug was originally marketed.

\section{CAVEATS}

\section{Specific study population may limit generalizability}

The DAPA-HF study included mostly male, White, European patients followed for an average of 18.2 months as part of initial Phase III studies funded by AstraZeneca (the pharmaceutical company that developed dapagliflozin). Given the potential conflict due to funding, all statistical results were verified by an independent academic group, and analyses were completed with an intention-totreat model. The outlined benefits described here were only studied in a population of patients with reduced $\mathrm{EF}(\leq 40 \%)$, so the impact remains unclear for patients with preserved EF. Safety and benefits beyond 24 months were not studied in this RCT; therefore longterm data are still unknown.

\section{CHALLENGES TO IMPLEMENTATION}

\section{Adding an SGLT2 inhibitor may be cost prohibitive for some patients}

An SGLT2 inhibitor costs, on average, $\$ 500$ to $\$ 600$ for a 30 -day supply, which may be prohibitive for some patients. ${ }^{11}$ Integration of SGLT2 inhibitors into a patient's medica- tion regimen may require dose adjustments of other medications, particularly glucoselowering therapies, and the optimal prioritization of medications is not yet known. JFP

\section{ACKNOWLEDGEMENT}

The PURLs Surveillance System was supported in part by Grant Number UL1RR024999 from the National Center For Research Resources, a Clinical Translational Science Award to the University of Chicago. The content is solely the responsibility of the authors and does not necessarily represent the official views of the National Center For Research Resources or the National Institutes of Health. Copyright @ 2021 . The Family Physicians Inquiries Network. All rights reserved.

\section{References}

1. McMurray JJV, Solomon SD, Inzucchi SE, et al. Dapagliflozin in patients with heart failure and reduced ejection fraction. $N$ Engl JMed. 2019;381:1995-2008. doi: 10.1056/NEJMoa1911303

2. Lytvyn Y, Bjornstad P, Udell JA, et al. Sodium glucose cotransporter-2 inhibition in heart failure: potential mechanisms, clinical applications, and summary of clinical trials. Circulation. 2017;136:1643-1658. doi: 10.1161/ CIRCULATIONAHA.117.030012

3. Virani SS, Alonso A, Benjamin EJ, et al. Heart disease and stroke statistics-2020 update: a report from the American Heart Association. Circulation. 2020;141:e139-e596. doi: 10.1161/ CIR.0000000000000757

4. Lloyd-Jones DM, Larson MG, Leip EP, et al. Lifetime risk for developing congestive heart failure: the Framingham Heart Study. Circulation. 2002;106:3068-3072. doi: 10.1161/ 01.cir.0000039105.49749.6

5. Ghosh RK, Ghosh GC, Gupta M, et al. Sodium glucose co-transporter 2 inhibitors and heart failure. Am J Cardiol. 2019;124 1790-1796. doi: 10.1016/j.amjcard.2019.08.038

6. Verma S, McMurray JJV. SGLT2 inhibitors and mechanisms of cardiovascular benefit: a state-of-the-art review. Diabetologia. 2018;61:2108-2117. doi: 10.1007/s00125-018-4670-7

7. Das SR, Everett BM, Birtcher KK, et al. 2020 expert consensus decision pathway on novel therapies for cardiovascular risk reduction in patients with type 2 diabetes: a report of the American College of Cardiology Solution Set Oversight Committee. J Am Coll Cardiol. 2020;76:1117-1145. doi: 10.1016/j.jacc.2020.05.037

8. Yancy CW, Jessup M, Bozkurt B, et al. 2013 ACCF/AHA guideline for the management of heart failure: a report of the American College of Cardiology Foundation/American Heart Association Task Force on Practice Guidelines. Circulation. 2013;128 e240-e327. doi: 10.1161/CIR.0b013e31829e8776

9. Nassif ME, Windsor SL, Tang F, et al. Dapagliflozin effects on biomarkers, symptoms, and functional status in patients with heart failure with reduced ejection fraction: The DEFINEHF Trial. Circulation. 2019;140:1463-1476. doi: 10.1161/ CIRCULATIONAHA.119.042929

10. Colucci WS. Secondary pharmacologic therapy in heart failure with reduced ejection fraction (HFrEF) in adults. UpToDate. Published October 9, 2020. Accessed June 23, 2021. www. uptodate.com/contents/secondary-pharmacologic-therapy-inheart-failure-with-reduced-ejection-fraction-hfref-in-adults

11. Dapagliflozin. GoodRx. Accessed June 23, 2021. www.goodrx. com/dapagliflozin
Integration of SGLT2 inhibitors into a patient's medication regimen may require dose adjustments of other medications. 